1 Hacettepe Journal of Mathematics and Statistics

$\bigcap$ Volume 45 (1) (2016), 121-128

\title{
Sharp results on linear combination of simple expressions of analytic functions
}

\author{
Nikola Tuneski*†, Teodor Bulboacă ${ }^{\ddagger}$ and Biljana Jolevska-Tuneska ${ }^{\S}$
}

\begin{abstract}
In this paper we use methods from the theory of differential subordinations to study the linear combination $a z f^{\prime \prime}(z)+b f^{\prime}(z)+c \frac{f(z)}{z}$ and give sharp bounds over the module, the argument and the real part of $\alpha f^{\prime}(z)+\beta \frac{f(z)}{z}$.
\end{abstract}

Keywords: analytic, univalent, linear combination, differential inequality, differential subordination.

2000 AMS Classification: Primary: 30C45, Secondary: 30C80.

Received: 10.03.2015 Accepted: 17.06.2015 Doi : 10.15672/HJMS.20164513114

\section{Introduction and preliminaries}

Let $H(\mathbb{D})$ denote the class of all analytic functions in the open unit disk $\mathbb{D}=\{z \in$ $\mathbb{C}:|z|<1\}$ and let $\mathcal{A}$ be the subclass of $H(\mathbb{D})$ consisting of those functions that are normalized with the condition $f(0)=f^{\prime}(0)-1=0$. The functions of $\mathcal{A}$ that are one-toone are called normalized univalent functions (for details see [4]).

A significant part of the theory of univalent functions deals with results over simple expressions of a function $f \in \mathcal{A}$ and its derivatives, such as

$$
z f^{\prime \prime}(z), \quad f^{\prime}(z) \text { and } \frac{f(z)}{z}
$$

and a small part of such results are presented in [6] and [2].

In this paper we will study the differential operator $\mathrm{I}(a, b, c): \mathcal{A} \rightarrow H(\mathbb{D})$ given by

$$
\mathrm{I}(a, b, c)[f](z):=a z f^{\prime \prime}(z)+b f^{\prime}(z)+c \frac{f(z)}{z}
$$

*Faculty of Mechanical Engineering, Ss. Cyril and Methodius University in Skopje, Karpoš II b.b., 1000 Skopje, Republic of Macedonia, Email: nikola.tuneski@mf .edu.mk

${ }^{\dagger}$ Corresponding Author.

${ }^{\ddagger}$ Faculty of Mathematics and Computer Science, Babeş-Bolyai University, 1, M. Kogălniceanu str., 400084 Cluj-Napoca, Romania, Email: bulboaca@math.ubbcluj.ro

${ }^{\S}$ Faculty of Electrical Engineering and Informational Technologies, Ss. Cyril and Methodius University in Skopje, Karpoš II b.b., 1000 Skopje, Republic of Macedonia,

Email: biljanaj@feit.ukim.edu.mk 
which is a linear combination of the above three expressions $z f^{\prime \prime}(z), f^{\prime}(z)$ and $f(z) / z$. Our results will provide simple sufficient conditions over the module, the argument and the real part of $\mathrm{I}(a, b, c)[f]$ that imply (in most of the cases) sharp bound of the module, the argument and the real part of another differential operator $\mathrm{J}(\alpha, \beta): \mathcal{A} \rightarrow H(\mathbb{D})$, given by

$$
\mathrm{J}(\alpha, \beta)[f](z):=\alpha f^{\prime}(z)+\beta \frac{f(z)}{z} .
$$

Special choice of the parameters $a, b$ and $c$ leads to numerous known results, and we remind some of them:

(i) the special case $a=c=0$ and $b=1$ brings results over the class of functions of bounded turning $[11,12,13]$;

(ii) the case $a=1$ and $b=c=0$ was studied in [9];

(iii) the case $b=1$ and $c=0$ was studied in [10];

(iv) the case $a=b=1$ and $c=0$ was studied in [3] and [1].

The sharpness of the results given in this paper closes, and in some cases improves and closes the related problems.

For our study we will use some methods from the theory of differential subordinations. Now we will recall the basic definitions and notion from this theory, that we need to use in our proofs. Valuable references on this topic may be found in [2] and [6].

If $f$ and $g$ are two analytic functions in the unit disk $\mathbb{D}$, then we say that $f$ is subordinate to $g$, written $f(z) \prec g(z)$, if there exists a Schwarz function $w$ (i.e. $w$ is analytic in $\mathbb{D}$ with $w(0)=0$ and $|w(z)|<1, z \in \mathbb{D})$ such that $f(z)=g(w(z))$ for all $z \in \mathbb{D}$. In particular, if $g$ is univalent in $\mathbb{D}$, then $f(z) \prec g(z)$ if and only if $f(0)=g(0)$ and $f(\mathbb{D}) \subseteq g(\mathbb{D})$.

The general theory of differential subordinations was introduced by Miller and Mocanu in [7] and [8]. Namely, if $\phi: \mathbb{C}^{2} \rightarrow \mathbb{C}$ is analytic in a domain $D \subset \mathbb{C}$, if $h$ is univalent in $\mathbb{D}$, and if $p$ is analytic in $\mathbb{D}$ with $\left(p(z), z p^{\prime}(z)\right) \in D$ for all $z \in \mathbb{D}$, then $p$ is said to satisfy a first-order differential subordination if

$$
\phi\left(p(z), z p^{\prime}(z)\right) \prec h(z) \text {. }
$$

A univalent function $q$ is said to be a dominant of the differential subordination (1.1) if $p(z) \prec q(z)$ for all the functions $p$ satisfying (1.1). If $\widetilde{q}$ is a dominant of (1.1) and $\widetilde{q}(z) \prec q(z)$ for all dominants of (1.1), then we say that $\widetilde{q}$ is the best dominant of the differential subordination (1.1).

To prove our main results we will use the following well-known lemmas from the theory of first-order differential subordinations:

1.1. Lemma. [8] Let $q$ be univalent in the unit disk $\mathbb{D}$, and let $\theta$ and $\phi$ be analytic in a domain $\mathcal{D}$ containing $q(\mathbb{D})$, with $\phi(w) \neq 0$ for all $w \in q(\mathbb{D})$. Set $Q(z)=z q^{\prime}(z) \phi(q(z))$, $h(z)=\theta(q(z))+Q(z)$, and suppose that

(i) $Q$ is starlike in the unit disk $\mathbb{D}$, and

(ii) $\operatorname{Re} \frac{z h^{\prime}(z)}{Q(z)}=\operatorname{Re}\left[\frac{\theta^{\prime}(q(z))}{\phi(q(z))}+\frac{z Q^{\prime}(z)}{Q(z)}\right]>0, z \in \mathbb{D}$.

If $p$ is analytic in $\mathbb{D}$, with $p(0)=q(0), p(\mathbb{D}) \subseteq \mathcal{D}$ and

$$
\theta(p(z))+z p^{\prime}(z) \phi(p(z)) \prec \theta(q(z))+z q^{\prime}(z) \phi(q(z))=h(z),
$$

then $p(z) \prec q(z)$, and $q$ is the best dominant of (1.2).

1.2. Lemma. [7, page 11] Let $n \geq 0$ be an integer and let $\gamma \in \mathbb{C}$, with $\operatorname{Re} \gamma>-n$. If $f(z)=\sum_{m \geq n} a_{m} z^{m}$ is analytic in $\mathbb{D}$ and $F$ is defined by

$$
F(z)=\frac{1}{z^{\gamma}} \int_{0}^{z} f(\zeta) \zeta^{\gamma-1} d \zeta=\int_{0}^{1} f(t z) t^{\gamma-1} d t
$$


then $F(z)=\sum_{m \geq n} \frac{a_{m} z^{m}}{m+\gamma}$ is analytic in $\mathbb{D}$.

Now, by using Lemma 1.1 and Lemma 1.2 we will prove

1.3. Lemma. Let $\alpha, \beta, \gamma \in \mathbb{C}$ with $\operatorname{Re} \gamma>-2$, and let $q$ be a univalent function in the unit disk $\mathbb{D}$, with $q(0)=\alpha+\beta$, satisfying

$$
\operatorname{Re}\left[1+\frac{z q^{\prime \prime}(z)}{q^{\prime}(z)}\right]>\max \{0,-1-\operatorname{Re} \gamma\}, z \in \mathbb{D} .
$$

(i) If $f \in \mathcal{A}$, then

$$
\mathrm{I}(\alpha, \alpha+\beta+\alpha \gamma, \beta \gamma)[f](z) \prec z q^{\prime}(z)+(1+\gamma) q(z)=: h(z),
$$

implies that

$$
\mathrm{J}(\alpha, \beta)[f](z) \prec q(z) .
$$

(ii) Moreover, if we suppose, in addition, that

$$
\operatorname{Re} \frac{\beta}{\alpha}>-2, \text { if } \alpha \beta \neq 0
$$

then the implication given in (i) is sharp. The extremal function $f_{*} \in \mathcal{A}$ that satisfies the subordination (1.4) such that $\mathrm{J}(\alpha, \beta)\left[f_{*}\right]=q$, is given by

$$
f_{*}(z)=\left\{\begin{array}{lll}
\frac{z q(z)}{\beta}, & \text { if } \alpha=0 \quad \text { and } \beta \neq 0, \\
z+\sum_{m \geq 1} \frac{q^{(m)}(0)}{(m+1) ! \alpha} z^{m+1}, & \text { if } \alpha \neq 0 \quad \text { and } \beta=0, \\
z+\sum_{m \geq 1} \frac{q^{(m)}(0)}{m !} \frac{z^{m+1}}{\beta+(m+1) \alpha}, & \text { if } \alpha \beta \neq 0 \quad \text { and } \quad \operatorname{Re} \frac{\beta}{\alpha}>-2 .
\end{array}\right.
$$

Proof. (i) Let define functions $\theta(w)=(1+\gamma) w$ and $\phi(w)=1, w \in \mathbb{C}$, that are analytic in the domain $\mathcal{D}=\mathbb{C}$ which contains $q(\mathbb{D})$, and $\phi(w) \neq 0$ for all $w \in q(\mathbb{D})$. Further, the condition (1.3) implies that for the functions $Q(z)=z q^{\prime}(z) \phi(q(z))=z q^{\prime}(z)$ and $h(z)=\theta(q(z))+Q(z)=(1+\gamma) q(z)+z q^{\prime}(z)$ we have

$$
\operatorname{Re} \frac{z Q^{\prime}(z)}{Q(z)}=\operatorname{Re}\left[1+\frac{z q^{\prime \prime}(z)}{q^{\prime}(z)}\right]>0, z \in \mathbb{D}
$$

and

$$
\operatorname{Re} \frac{z h^{\prime}(z)}{Q(z)}=\operatorname{Re}\left[2+\gamma+\frac{z q^{\prime \prime}(z)}{q^{\prime}(z)}\right]=1+\operatorname{Re} \gamma+\operatorname{Re}\left[1+\frac{z q^{\prime \prime}(z)}{q^{\prime}(z)}\right]>0, z \in \mathbb{D} .
$$

So, the conditions (i) and (ii) from Lemma 1.1 hold, and moreover, the function $h$ is close-to-convex, hence univalent in $\mathbb{D}$ (see [5]).

Let now choose $p(z)=\mathrm{J}(\alpha, \beta)[f](z)=\alpha f^{\prime}(z)+\beta \frac{f(z)}{z}$. Then, the function $p$ is analytic in $\mathbb{D}$, with $p(0)=q(0)=\alpha+\beta$, and $p(\mathbb{D}) \subseteq \mathcal{D}=\mathbb{C}$. Finally, bearing in mind that

$$
\mathrm{I}(\alpha, \alpha+\beta+\alpha \gamma, \beta \gamma)[f](z)=z p^{\prime}(z)+(1+\gamma) p(z)
$$

we obtain that the subordinations (1.2) and (1.4) are equivalent, and the conclusion follows immediately from Lemma 1.1.

(ii) We will prove the second part of our lemma by showing that the differential equation

$$
\alpha f^{\prime}(z)+\beta \frac{f(z)}{z}=q(z)
$$

has a solution $f_{*} \in \mathcal{A}$, whenever the assumptions (a) and (b) hold. For this purpose we will divide the analysis in three different cases. 
Case 1. If $\alpha=0$ and $\beta \neq 0$, since $q(0)=\alpha+\beta=\beta$, it follows that the equation (1.6) has solution $f_{*}(z)=\frac{z q(z)}{\beta} \in \mathcal{A}$.

Case 2. If $\alpha \neq 0$ and $\beta=0$, then (1.6) has the analytic solution

$$
f_{*}(z)=\frac{1}{\alpha} \int_{0}^{z} q(\zeta) d \zeta=z+\sum_{m \geq 1} \frac{q^{(m)}(0)}{(m+1) ! \alpha} z^{m+1},
$$

and since $q(0)=\alpha+\beta=\alpha$ the above function $f_{*}$ belongs to $\mathcal{A}$.

Case 3. Now let analyse the more complex case, assuming that $\alpha \beta \neq 0$. The equation (1.6) is equivalent to

$$
f(z)+\frac{1}{\Gamma} z f^{\prime}(z)=H(z), \quad \text { where } \quad \Gamma:=\frac{\beta}{\alpha} \quad \text { and } \quad H(z):=\frac{z q(z)}{\beta} .
$$

It is easy to see that

$$
H(z)=\sum_{m \geq 1} a_{m} z^{m}, z \in \mathbb{D}, \quad \text { where } \quad a_{m}=\frac{q^{(m-1)}(0)}{(m-1) ! \beta} .
$$

Since the function $q$ is univalent in $\mathbb{D}$, and thus $q^{\prime}(z) \neq 0$ for all $z \in \mathbb{D}$, it follows that the second coefficient of the above power expansion of $H$ does not vanish, i.e. $\frac{q^{\prime}(0)}{\beta} \neq 0$.

Further, according to Lemma 1.2, the differential equation (1.7) has the analytic solution

$$
f_{*}(z)=\frac{\Gamma}{z^{\Gamma}} \int_{0}^{z} H(\zeta) \zeta^{\Gamma-1} d \zeta=\frac{1}{\alpha z^{\beta / \alpha}} \int_{0}^{z} q(\zeta) \zeta^{\beta / \alpha} d \zeta, z \in \mathbb{D}
$$

whenever $\operatorname{Re} \Gamma>-n$

This means that the solution (1.8) of the differential equation (1.6) is analytic in $\mathbb{D}$ if we assume that

$$
\operatorname{Re} \Gamma=\operatorname{Re} \frac{\beta}{\alpha}>-2 .
$$

Hence, the solution (1.8) of the differential equation (1.6) is an analytic function in $\mathbb{D}$, and has the form

$$
f_{*}(z)=z+\sum_{m \geq 1} \frac{q^{(m)}(0)}{m !} \frac{z^{m+1}}{\beta+(m+1) \alpha}, z \in \mathbb{D}
$$

that is $f_{*} \in \mathcal{A}$.

\section{Results over the module}

In this section we study the module of $\mathrm{I}(a, b, c)[f]$ and receive sharp information about the module of $\mathrm{J}(\alpha, \beta)[f]$.

2.1. Theorem. Let $\alpha, \beta, \gamma \in \mathbb{C}$ with $\beta+2 \alpha \neq 0$, $\operatorname{Re} \gamma>-2$, and let $\delta>0$. If $f \in \mathcal{A}$, then

$$
|\mathrm{I}(\alpha, \alpha+\beta+\alpha \gamma, \beta \gamma)[f](z)-(1+\gamma)(\alpha+\beta)|<\delta, z \in \mathbb{D}
$$

implies

$$
|\mathrm{J}(\alpha, \beta)[f](z)-(\alpha+\beta)|<\Delta=\Delta(\gamma, \delta):=\frac{\delta}{|2+\gamma|}, z \in \mathbb{D} .
$$

This implication is sharp, and the extremal function is

$$
f_{*}(z)=z+\frac{\delta}{(2+\gamma)(\beta+2 \alpha)} z^{2} .
$$


Proof. If we denote $q(z)=\alpha+\beta+\frac{\delta}{2+\gamma} z, z \in \mathbb{D}$, then we have $1+\frac{z q^{\prime \prime}(z)}{q^{\prime}(z)}=1$, meaning that (1.3) from Lemma 1.3 holds because of the assumption $\operatorname{Re} \gamma>-2$. Further, the function $h$ defined in (1.4) will be

$$
h(z)=(1+\gamma)(\alpha+\beta)+\delta z, z \in \mathbb{D},
$$

hence the subordination (1.4) is equivalent to (2.1). Therefore, (2.2) follows directly from Lemma 1.3 and the definition of subordination, while a simple computation shows that $f_{*}$ given by $(2.3)$ is the extremal function.

If we consider in the above theorem the special case $\alpha=-\beta=1$, then we obtain the first part, while for $\beta=1-\alpha$ we obtain the second part of the next corollary:

2.2. Corollary. Let $\delta>0$ and $\gamma \in \mathbb{C}$, with $\operatorname{Re} \gamma>-2$.

(i) If $f \in \mathcal{A}$, then

$$
\left|z f^{\prime \prime}(z)+\gamma f^{\prime}(z)-\gamma \frac{f(z)}{z}\right|<\delta, z \in \mathbb{D}
$$

implies

$$
\left|f^{\prime}(z)-\frac{f(z)}{z}\right|<\Delta=\Delta(\gamma, \delta), z \in \mathbb{D} .
$$

This implication is sharp, and the extremal function is $f_{*}(z)=z+\frac{\delta}{2+\gamma} z^{2}$.

(ii) Assuming that $\alpha \in \mathbb{C} \backslash\{-1\}$, if $f \in \mathcal{A}$, then

$$
\left|\alpha z f^{\prime \prime}(z)+(1+\alpha \gamma) f^{\prime}(z)+(1-\alpha) \gamma \frac{f(z)}{z}-(1+\gamma)\right|<\delta, z \in \mathbb{D},
$$

implies

$$
\left|\alpha f^{\prime}(z)+(1-\alpha) \frac{f(z)}{z}-1\right|<\Delta=\Delta(\gamma, \delta), z \in \mathbb{D} .
$$

This implication is sharp, and the extremal function is $f_{*}(z)=z+\frac{\delta}{(2+\gamma)(\alpha+1)} z^{2}$.

\section{Results over the argument and the real part}

In this section we study the argument of the $\mathrm{I}(\alpha, \alpha+\beta+\alpha \gamma, \beta \gamma)[f]$ and obtain a result for the argument of $\mathrm{J}(\alpha, \beta)[f]$. As an interesting consequence, we receive the corresponding result over the real parts of these operators.

3.1. Theorem. Let $\alpha, \beta \in \mathbb{C}$ and $\gamma, \lambda \in \mathbb{R}$, such that $\alpha+\beta>\lambda, \gamma \geq-1$, and also let $\theta \in(0,1]$.

(i) If $f \in \mathcal{A}$, then

$$
|\arg [\mathrm{I}(\alpha, \alpha+\beta+\alpha \gamma, \beta \gamma)[f](z)-(1+\gamma) \lambda]|<\Delta, z \in \mathbb{D},
$$

where

$$
\Delta=\Delta(\theta, \gamma):= \begin{cases}\frac{\theta \pi}{2}+\arctan \frac{\theta}{1+\gamma}, & \text { if } \gamma>-1 \\ \frac{(\theta+1) \pi}{2}, & \text { if } \gamma=-1\end{cases}
$$

implies

$$
|\arg \mathrm{J}(\alpha, \beta)[f](z)-\lambda|<\frac{\theta \pi}{2}, z \in \mathbb{D} .
$$


(ii) Moreover, for the special case $\gamma=-1$, if we suppose, in addition, that

$$
\operatorname{Re} \frac{\beta}{\alpha}>-2, \text { if } \alpha \beta \neq 0 \text {, }
$$

then the implication given in (i) is sharp.

Proof. The assumption $\alpha+\beta>\lambda$, with $\lambda \in \mathbb{R}$, holds if and only if $\operatorname{Im} \alpha=-\operatorname{Im} \beta$ and $\operatorname{Re} \alpha+\operatorname{Re} \beta>\lambda$.

Let consider the function $q(z)=\left(\frac{1+z}{1-z}\right)^{\theta}(\operatorname{Re} \alpha+\operatorname{Re} \beta-\lambda)+\lambda, z \in \mathbb{D}$, where the power is taken to its principal value. It follows that $q(0)=\operatorname{Re} \alpha+\operatorname{Re} \beta$,

$$
1+\frac{z q^{\prime \prime}(z)}{q^{\prime}(z)}=: H(z)=-1+\frac{2(1+\theta z)}{1-z^{2}}, z \in \mathbb{D}
$$

hence

$$
H\left(e^{i \varphi}\right)=i \frac{\theta+\cos \varphi}{\sin \varphi}, \varphi \in(-\pi, 0) \cup(0, \pi) .
$$

Since $H(0)=1$, we deduce $\operatorname{Re} H(z)>0, z \in \mathbb{D}$, and from $q^{\prime}(0)=2 \theta(\operatorname{Re} \alpha+\operatorname{Re} \beta-\lambda) \neq$ 0 we conclude that $q$ is a convex (univalent) function in $\mathbb{D}$.

Thus, the initial assumptions of Lemma 1.3 are satisfied. The proof will be completed if we show that the inequality (3.1) implies the subordination (1.4), and the subordination (1.5) is equivalent to the inequality (3.2).

The function $h$ defined in the subordination (1.4) has the form

$h(z)=z q^{\prime}(z)+(1+\gamma) q(z)=(\operatorname{Re} \alpha+\operatorname{Re} \beta-\lambda)\left(\frac{1+z}{1-z}\right)^{\theta}\left(\frac{2 \theta z}{1-z^{2}}+1+\gamma\right)+(1+\gamma) \lambda$.

Even more, since $(h(z)-h(0)) / h^{\prime}(0)$ is a close-to-convex (normalized) function in $\mathbb{D}$, it follows that the function $h$ is univalent.

Now, for $z=e^{i \varphi}$ and $\varphi \in(-\pi, 0) \cup(0, \pi)$, using the fact that $\alpha+\beta>\lambda$ we get

$$
\begin{gathered}
\arg \left[h\left(e^{i \varphi}\right)-(1+\gamma) \lambda\right]=\arg \left[\left(\frac{1+e^{i \varphi}}{1-e^{i \varphi}}\right)^{\theta}\left(\frac{2 \theta e^{i \varphi}}{1-e^{2 i \varphi}}+1+\gamma\right)\right] \\
=\arg \left[\left(i \cot \frac{\varphi}{2}\right)^{\theta}\left(\frac{i \theta}{\sin \varphi}+1+\gamma\right)\right],
\end{gathered}
$$

hence

$$
\arg \left[h\left(e^{i \varphi}\right)-(1+\gamma) \lambda\right]=\theta \arg \left(i \cot \frac{\varphi}{2}\right)+\arg \left(\frac{i \theta}{\sin \varphi}+1+\gamma\right) .
$$

We will discuss now the following two cases.

Case 1. If $\gamma>-1$, from the relation (3.3) we easily deduce that

$$
\arg \left[h\left(e^{i \varphi}\right)-(1+\gamma) \lambda\right] \geq \frac{\theta \pi}{2}+\arctan \frac{\theta}{1+\gamma}, \text { if } \varphi \in(0, \pi)
$$

and

$$
\arg \left[h\left(e^{i \varphi}\right)-(1+\gamma) \lambda\right] \leq-\left(\frac{\theta \pi}{2}+\arctan \frac{\theta}{1+\gamma}\right), \text { if } \varphi \in(-\pi, 0),
$$

which implies

$$
\left|\arg \left[h\left(e^{i \varphi}\right)-(1+\gamma) \lambda\right]\right| \geq \frac{\theta \pi}{2}+\arctan \frac{\theta}{1+\gamma}, \text { for } \varphi \in(-\pi, 0) \cup(0, \pi) .
$$

These inequalities, combined with the fact that $h(0)=(\operatorname{Re} \alpha+\operatorname{Re} \beta)(1+\gamma)>(1+\gamma) \lambda$ and the fact that $h$ is univalent in $\mathbb{D}$, leads to the conclusion that

$$
\Omega:=\left\{w \in \mathbb{C}:|\arg [w-(1+\gamma) \lambda]|<\frac{\theta \pi}{2}+\arctan \frac{\theta}{1+\gamma}\right\} \subset h(\mathbb{D}),
$$


and from (3.3) we may see that

$$
\left|\arg \left[h\left(e^{i \varphi}\right)-(1+\gamma) \lambda\right]\right|=\frac{\theta \pi}{2}+\arctan \frac{\theta}{1+\gamma} \Leftrightarrow \varphi \in\left\{-\frac{\pi}{2}, \frac{\pi}{2}\right\},
$$

hence $\Omega \neq h(\mathbb{D})$.

The assumption (3.1) is equivalent to $\mathrm{I}(\alpha, \alpha+\beta+\alpha \gamma, \beta \gamma)[f](\mathbb{D}) \subset \Omega$, and according to (3.4) this inclusion implies $\mathrm{I}(\alpha, \alpha+\beta+\alpha \gamma, \beta \gamma)[f](\mathbb{D}) \subset h(\mathbb{D})$. Using the fact that $\mathrm{I}(\alpha, \alpha+\beta+\alpha \gamma, \beta \gamma)[f](0)=h(0)=(\operatorname{Re} \alpha+\operatorname{Re} \beta)(1+\gamma)$, this inclusion is equivalent to the subordination (1.4), and by Lemma 1.3 if follows that $\mathrm{J}(\alpha, \beta)[f](z) \prec q(z)$, which is equivalent to our conclusion (3.2).

Case 2. If $\gamma=-1$, from the relation (3.3) we similarly deduce that the conclusion is even stronger, that is

$$
h(\mathbb{D})=\left\{w \in \mathbb{C}:|\arg w|<\frac{(\theta+1) \pi}{2}\right\} .
$$

In this case, the assumption (3.1) is equivalent to $\mathrm{I}(\alpha, \alpha+\beta+\alpha \gamma, \beta \gamma)[f](\mathbb{D})=h(\mathbb{D})$, and from $\mathrm{I}(\alpha, \alpha+\beta+\alpha \gamma, \beta \gamma)[f](0)=h(0)=(\operatorname{Re} \alpha+\operatorname{Re} \beta)(1+\gamma)$, the previous equality between the two sets is equivalent to the subordination (1.4). Thus, from Lemma 1.3 it follows that $\mathrm{J}(\alpha, \beta)[f](z) \prec q(z)$, which is equivalent to our conclusion (3.2).

Notice that, for $\gamma=-1$ the result is sharp. That's because the assumption (3.1) is equivalent to the subordination (1.4), while in the case $\gamma>-1$ the assumption (3.1) is stronger than (1.4).

By specifying values of some of the parameters in Theorem 3.1 we receive the next results:

3.2. Corollary. Let $\alpha, \beta \in \mathbb{C}$ and $\gamma, \lambda \in \mathbb{R}$, such that $\alpha+\beta>\lambda, \gamma \geq-1$.

(i) If $f \in \mathcal{A}$, then

$$
\operatorname{Re}\left[\alpha z f^{\prime \prime}(z)+(\alpha+\beta+\alpha \gamma) f^{\prime}(z)+\beta \gamma \frac{f(z)}{z}\right]>(1+\gamma) \lambda, z \in \mathbb{D},
$$

implies

$$
\operatorname{Re}\left[\alpha f^{\prime}(z)+\beta \frac{f(z)}{z}\right]>\lambda, z \in \mathbb{D} .
$$

Moreover, for the special case $\gamma=-1$, if we suppose, in addition, that

$$
\text { (a) } \operatorname{Re} \frac{\beta}{\alpha}>-2 \text {, if } \alpha \beta \neq 0 \text {, }
$$

then the implication given in (i) is sharp.

(ii) Assuming that $\lambda<0$, if $f \in \mathcal{A}$, then

$$
\operatorname{Re}\left[z f^{\prime \prime}(z)+\gamma f^{\prime}(z)-\gamma \frac{f(z)}{z}\right]>(1+\gamma) \lambda, z \in \mathbb{D}
$$

implies

$$
\operatorname{Re}\left[f^{\prime}(z)-\frac{f(z)}{z}\right]>\lambda, z \in \mathbb{D} \text {. }
$$

Moreover, for the special case $\gamma=-1$ the implication given in (ii) is sharp.

(iii) Assuming that and $\lambda<1$, if $f \in \mathcal{A}$, then

$$
\operatorname{Re}\left[\alpha z f^{\prime \prime}(z)+(1+\alpha \gamma) f^{\prime}(z)+(1-\alpha) \gamma \frac{f(z)}{z}\right]>(1+\gamma) \lambda, z \in \mathbb{D},
$$

implies

$$
\operatorname{Re}\left[\alpha f^{\prime}(z)+(1-\alpha) \frac{f(z)}{z}\right]>\lambda, z \in \mathbb{D} \text {. }
$$


Moreover, for the special case $\gamma=-1$, if we suppose, in addition, that

$$
\left(a^{\prime}\right) \quad\left|\alpha+\frac{1}{2}\right|>\frac{1}{2} \quad \text { if } \quad \alpha \notin\{0 ; 1\},
$$

then the implication given in (iii) is sharp.

Proof. The implication (i) follows directly from Theorem 3.1 for $\theta=1$ having in mind that $\frac{\pi}{2}+\arctan \frac{1}{1+\gamma}>\frac{\pi}{2}$. The implication (iii) follows from (i) if we choose $\alpha+\beta=1$, and in that case the assumption (a) is equivalent to (a'). The implication (ii) was obtained from (i) for the special case $\alpha=-\beta=1$.

3.3. Remark. Taking $\alpha=1$ and $\beta=\gamma=0$ in Corollary 3.2(i), we receive that for $\lambda<1$ and $f \in \mathcal{A}$ the following implication holds:

$$
\operatorname{Re}\left[z f^{\prime \prime}(z)+f^{\prime}(z)\right]>\lambda, z \in \mathbb{D} \Rightarrow \operatorname{Re} f^{\prime}(z)>\lambda, z \in \mathbb{D} .
$$

This improves the result given in Theorem 1(a) from [1] where it was proven that

$$
\operatorname{Re}\left[z f^{\prime \prime}(z)+f^{\prime}(z)\right]>\lambda, z \in \mathbb{D} \Rightarrow \operatorname{Re} f^{\prime}(z)>1+2(1-\lambda)(\log 2-1), z \in \mathbb{D} .
$$

Implication (3.5) is stronger than the implication (3.6), since for $\lambda<1$ we have

$$
1+2(1-\lambda)(\log 2-1)<\lambda \text {. }
$$

Acknowledgment. The work of the second author was entirely supported by the grant given by Babeş-Bolyai University dedicated for Supporting the Excellence Research 2015.

\section{References}

[1] Ali R. M., On a subclass of starlike functions, Rocky Mountain J. Math. 24 (1994), no. 2, 447-451.

[2] Bulboacă T., Differential subordinations and superordinations. New results, House of Science Book Publ., Cluj-Napoca, 2005.

[3] Chichra P. N., New subclasses of the class of close-to-convex functions, Proc. Amer. Math. Soc. 62 (1977), 37-43.

[4] Duren P. L., Univalent functions, Springer-Verlag, 1983.

[5] Kaplan W., Close to convex schlicht functions, Michig. Math. J. 1 (1952), no. 2, 169-185.

[6] Miller S. S., Mocanu P. T., Differential subordinations, Theory and Applications, Marcel Dekker, New York-Basel, 2000.

[7] Miller S. S., Mocanu P. T., Differential subordinations and univalent functions, Michigan Math. J. 28 (1981), 157-171.

[8] Miller S. S., Mocanu P. T., On some classes of first-order differential subordinations, Michigan Math. J. 32 (1985), 185-195.

[9] Obradović M., Simple sufficient conditions for starlikeness, Mat. Vesnik 49 (1997), 241-244.

[10] Ponnusamy S., Singh V., Criteria for univalent, starlike and convex functions, Bull. Belg. Math. Soc. Simon Stevin 9 (2002), no. 4, 511-531.

[11] Sokol J., Some applications of differential subordinations in the geometric function theory, J. Inequal. Appl. 2013, 2013:389, 11 pp.

[12] Tuneski N., Some simple sufficient conditions for starlikeness and convexity, Appl. Math. Lett. 22 (2009), 693-697.

[13] Tuneski N., Obradović M., Some properties of certain expression of analytic functions, Comput. Math. Appl. 62 (2011), 3438-3445. 